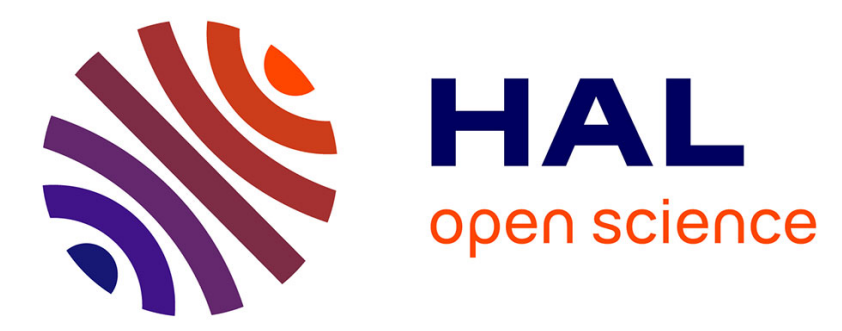

\title{
Near surface soil moisture estimation from microwave measurements
}

\author{
Laurent Bruckler, H. Witono, Pierre Stengel
}

\section{To cite this version:}

Laurent Bruckler, H. Witono, Pierre Stengel. Near surface soil moisture estimation from microwave measurements. Remote Sensing of Environment, 1988, 26, pp.101-121. hal-02727425

\section{HAL Id: hal-02727425 \\ https://hal.inrae.fr/hal-02727425}

Submitted on 2 Jun 2020

HAL is a multi-disciplinary open access archive for the deposit and dissemination of scientific research documents, whether they are published or not. The documents may come from teaching and research institutions in France or abroad, or from public or private research centers.
L'archive ouverte pluridisciplinaire HAL, est destinée au dépôt et à la diffusion de documents scientifiques de niveau recherche, publiés ou non, émanant des établissements d'enseignement et de recherche français ou étrangers, des laboratoires publics ou privés. 


\title{
Near Surface Soil Moisture Estimation from Microwave Measurements
}

\author{
L. BRUCKLER, ${ }^{*}$ H. WTTONO ${ }^{\dagger}$ AND P. STENGEL* \\ *INRA Station de Science du Sol, B.P. 91, Domaine Saint Paul, 84140 Montfavet, France \\ 'Department of Soil Science, Bogor Agricultural University, J1. Raya Pajajaran, Bogor, Indonesia
}

\begin{abstract}
Soil moisture, water potential, and bulk density measurements were performed on a 0.4 ha bare field $(2 \% .2 \%$ clay, $61.7^{\circ}$ fine and coarse loam) concurrent with backscattering coefficient measurements: 17 different volumetric water content profiles from 0 to $10 \mathrm{~cm}$ were sampled including wet, intermediate, and dry conditions. Backscattering coefficients were measured using a $4.5 \mathrm{GHz}$ frequency, $\mathrm{HH}$ polarization and $15-20^{\circ}$ incidence angle microwave sensor configuration, in order to minimize the soil surface roughness effects. First, the statistical analysis of the data ("classical calibration procedure") exhibited satisfactory regression lines between the backscattering coefficient and the volumetric water content calculated over arbitrary soil depths, as described by many authors (correlation coefficients between 0.859 and 0.899 ). Furthermore, the same results were obtained when water potential data were considered (log scale). Second, taking into account the dependence between the water content profile and the microwave penetration depth, the experimental relationship betveen backscattering coefficient and volumetric water content became nonlinear and exhibited smaller residuals than the "classical regression line." Finally, a statistical procedure for predicting the mean and standard deviation of volumetric water content profiles from the soil surface to the microwave penetration depth is presented. Results showed that the proposed procedure is promising for obtaining near-surface water content estimates used as boundary conditions for "soil/atmosphere" water transport modeling.
\end{abstract}

\section{Introduction}

Extensive studies have been performed in the last years by several researchers using active microwave sensors in order i) to test the capability of such equipment to measure the soil surface water content (Bernard et al., 1982; Bradley and Ulaby, 1981; Hirosawa et al., 1978; Jackson and O'Neill, 1985; Le Toan et al., 1981; Pausader, 1982; Ulaby, 1974; Ulaby et al., 1978) and ii) to monitor the water budget at a field or regional scale (Bernard et al., 1981; Prevot et al., 1984).

An optimal active microwave sensor configuration for soil moisture measurements has been established: It was shown that, for a radar operating near $5 \mathrm{GHz}$, $\mathrm{HH}$ polarization, at incidence angles between $7^{\circ}$ and $17^{\circ}$, the backscattering coefficient was correlated to soil volu- metric water content, and quite high correlation coefficients between soil water metric potential and backscattering coefficients were found over a relatively small water tension range (Dobson and Ulaby, 1981). Furthermore, using this optimal combination of frequency, look angle, and polarization, measurements exhibited a minimal dependence on soil surface roughness characteristics.

Attempts were made to use microwaves measurements for water budget monitoring: Bernard et al. (1981) provided numerical computations on the basis of water transfer deterministic model in order to demonstrate that such measurements can be used as boundary conditions at the soil surface. Bernard et al. (1986) used a more empirical approach to compare soil hydrodynamic properties between different fields by microwave 
measurements. The results were encouraging and indicated the possible use of microwave sensors to provide boundary conditions for controlling soil surface evaporation and water storage at field scale.

In most studies on microwave measurements on bare soils, experimental relationships between soil moisture content and backscattering coefficient were provided by mean volumetric water contents measured from the soil surface to an arbitrary soil depth, generally $0-5$ or $0-10$ $\mathrm{cm}$. In fact, soil moisture or water potential gradients are sometimes very high in the top thin soil layers, which makes it difficult to attribute a real physical sense to calculated moisture content means. Furthermore, soil water movements between soil surface and atmospheric boundary layers exhibit a greater sensitivity to water content or water potential just at the soil surface than to the mean water content in the top soil layers. Thus, since the shape of the water content profile varies widely under natural conditions, this is probably an actual limiting factor of remote sensing measurements to provide precise soil surface boundary conditions for modeling water movements between soil and atmosphere.

This paper is an experimental contribution for evaluating the effect of soil moisture gradients near the soil surface on backscattering coefficient measurements: In the first part, we present empirical results which show classical relationships between soil moisture content or water potential and backscattering coefficient measurements ("classical calibration procedure"). In the second part, we propose to test if taking into account the calculated "microwave penetration depth" is able to provide a more realistic and physically based regression curve between the backscattering coefficient and the soil moisture content. Finally, we describe a statistical approach for estimating soil water profiles from the soil surface to the maximum microwave penetration depth using microwave measurements in order to provide accurate estimates of the water content at the soil surface.

\section{Materials and Methods: \\ Soil and Backscattering \\ Coefficient Measurements}

Experiments were carried out on a bare field $(0.4 \mathrm{ha})$ located at Montfavet (France), using a scatterometer mounted on a mobile platform. Ground observations of soil moisture and dry bulk density concurrent with scatterometer measurements were performed on the experimental field which was composed of $27.2 \%$ clay and $61.7 \%$ loam.

Precise gravimetric soil moisture measurements were collected for 17 days using irrigation equipment in order to obtain wet, intermediate and dry conditions. For each day, 12-18 individual water content profiles from 0 to $10 \mathrm{~cm}$, $(0-1 ; 1-2 ; 2-3 ; 3-4 ; 4-5 ; 5-6 ; 6-7$; and $7-10 \mathrm{~cm}$ ) were sampled to estimate the mean water content at field scale. To insure precise measurements for each depth, soil surface roughness was chosen as low as possible (RMS height variation $\leqslant 1 \mathrm{~cm}$ ). Furthermore, a preliminary geostatistical analysis was conducted on the field to insure a statistical independence between the water content measurements for each sampling location. Water potentials were measured during the experiment using i) tensiometers, ii) pressure plates for water potential between -1 and -16 bars and, iii) laboratory vapor 
equilibrium device for 15-30 days for very dry soil samples. Thus, water potential was measured for wet to very dry soil conditions, and a small variability between replicates was observed $(\leqslant 0.01$ $\mathrm{cm}^{3} / \mathrm{cm}^{3}$ ).

Dry bulk density was measured using a field gamma-ray transmission apparatus having a precision of $0.02-0.03 \mathrm{~g} / \mathrm{cm}^{3}$ for thin soil layers (Bertuzzi et al., 1987). Volumetric water content profiles were easily estimated by combining gravimetric soil moisture and dry bulk density measurements.

The radar was mounted on a mobile platform (altitude $14 \mathrm{~m}$ ) moving on a railway $(100 \mathrm{~m})$. Based on previous studies, measurements were performed for 4.5 $\mathrm{GHz}$ frequency, $15^{\circ}$ or $20^{\circ}$ look angle, and $\mathrm{HH}$ polarization, and two or three replicates of microwave measurements were made all over the experimental field during soil water sampling.

\section{Theory}

Several attempts have been made to provide more or less precise microwave penetration models, including effects due to simple soil surface reflection or multireflection within the soil layers (Pausader, 1982). In order to analyze the relationship between soil water content profile and microwave penetration depth, let us summarize the main steps of the theoretical calculations: The air is regarded as a real medium and the soil as a complex one. We assume that the soil surface is a infinite plane, and that the soil depth is divided into $n$ thin soil layers: At each soil layer boundary, the incident wave amplitude is i) partly transmitted and ii) partly attenuated within each layer. It is to be noted that only one reflection is taken into account at each boundary between two different layers.

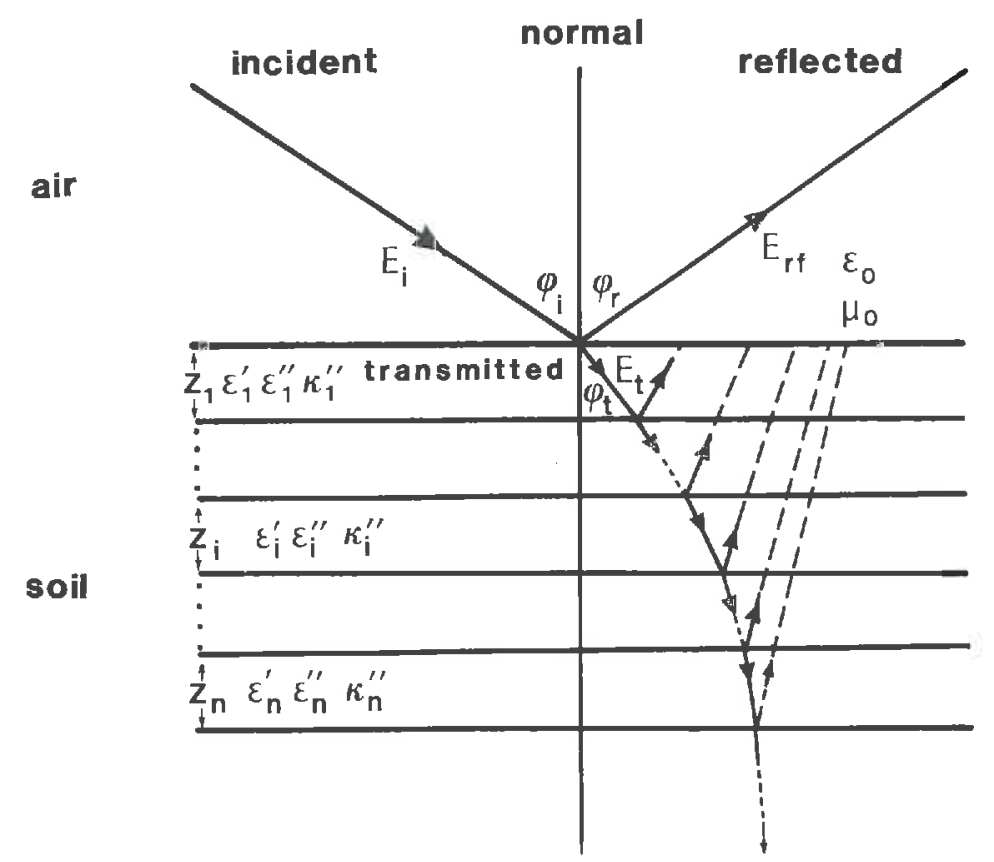

FIGURE 1. Schematic representation of incident and transmitted microwaves in soil layers. 
In the text, the subscripts $i$ and $t$ refer to the "incident layer" and to the "transmission layer," respectively (Fig. 1). The subscript $r$ corresponds to the term "relative," which is used for permittivity calculations. If the incident medium is characterized by a real refraction index $\left(n_{i}\right)$ and an incidence angle $\left(\theta_{i}\right)$, and if the transmission medium has a complex refraction index $\left(n_{t}\right)$ and a complex refraction angle $\left(\theta_{t}\right)$, the basic SnellDescartes law gives

$$
n_{i} \cdot \sin \theta_{i}=n_{t} \cdot \sin \theta_{t}
$$

After Becker (1978), this makes it possible to modify Eq. (1), considering that the complex transmission medium is equivalent to a "real medium" having a "real equivalent refraction index" given by $\left[\mu\left(\theta_{t}^{\prime}\right)\right]$ and a "real refraction angle" $\left(\theta_{t}^{\prime}\right)$. Thus, $\mu$ and $\theta^{\prime}$ are defined as follows:

$$
n_{t} \cdot \sin \theta_{t}=\mu\left(\theta_{t}^{\prime}\right) \cdot \sin \theta_{t}^{\prime}
$$

Following Becker (1978), the real terms $\left[\mu\left(\theta_{t}^{\prime}\right)\right]$ and $\left(\theta_{t}^{\prime}\right)$ are given in terms of real and imaginary parts of the refraction index $\left(n_{t}^{\prime}, n_{t}^{\prime \prime}\right)$. It is more convenient for further calculations to write these terms using the real and imaginary parts of the complex permittivity for the transmission medium $\left(\varepsilon_{r t}^{\prime}, \varepsilon_{r t}^{\prime \prime}\right)$.

Given that

$$
\left[\mu\left(\theta_{t}^{\prime}\right)\right]^{2}=\Gamma_{r t}
$$

where $\left(\Gamma_{r t}\right)$ is the real permittivity for the "equivalent medium," we obtain

$$
\begin{aligned}
\Gamma_{r t} & =\frac{1}{2 \cdot \cos ^{2} \theta_{t}^{\prime}}\left[\left(\varepsilon_{r t}^{\prime} \cdot \cos ^{2} \theta_{t}^{\prime}\right)\right. \\
& \left.+\left(\varepsilon_{r t}^{\prime 2} \cdot \cos ^{4} \theta_{t}^{\prime}+\varepsilon_{r t}^{\prime \prime 2} \cdot \cos ^{2} \theta_{t}^{\prime}\right)^{1 / 2}\right]
\end{aligned}
$$

and, for the $\left(\theta_{t}^{\prime}\right)$ angle,

$$
\sin ^{2} \theta_{t}^{\prime}=\frac{f_{1}\left(\varepsilon_{r t}^{\prime}, \varepsilon_{r t}^{\prime \prime}\right)}{f_{2}\left(\varepsilon_{r t}^{\prime}, \varepsilon_{r t}^{\prime \prime}\right)},
$$

where

$$
\begin{array}{r}
f_{1}=\varepsilon_{r i} \cdot \sin ^{2} \theta_{i} \cdot\left\{\left(-\varepsilon_{r t}^{\prime}-\varepsilon_{r i} \cdot \sin ^{2} \theta_{i}\right)\right. \\
+\left[\left(\varepsilon_{r t}^{\prime 2}+\varepsilon_{r t}^{\prime \prime 2}\right)+\varepsilon_{r i}^{2} \cdot \sin ^{4} \theta_{i}\right. \\
\left.\left.-2 \cdot \varepsilon_{r i} \cdot \sin ^{2} \theta_{i} \cdot\left(-\varepsilon_{r t}^{\prime}\right)\right]^{1 / 2}\right\}
\end{array}
$$

and

$$
f_{2}=2 \cdot\left[\left(\varepsilon_{r t}^{\prime \prime 2} / 4\right)-\varepsilon_{r i} \cdot \sin ^{2} \theta_{i} \cdot \varepsilon_{r t}^{\prime}\right]
$$

Thus, the transmission coefficient $\left(T_{i, t}\right)$ from a real incident medium to a real refraction medium may be calculated using

$$
T_{i, t}=\frac{2 \cdot\left(\varepsilon_{r i}\right)^{1 / 2} \cdot \cos \theta_{i}}{\left(\varepsilon_{r i}\right)^{1 / 2} \cdot \cos \theta_{i}+\left(\Gamma_{r t}\right)^{1 / 2} \cdot \cos \theta_{t}^{\prime}}
$$

where $\left(\varepsilon_{r i}\right)$ is the real relative dielectric permittivity of the incident medium, $\left(\Gamma_{r t}\right)$ the real relative dielectric permittivity of the refraction medium given by Eq. (4), and $\left(\theta_{t}^{\prime}\right)$ is the real refraction angle defined by Eqs. (5) $-(7)$.

The attenuation coefficient for any soil layer is given by the imaginary part of the wave vector $\left(k^{\prime \prime}\right)$ :

$$
\begin{aligned}
k^{\prime \prime}= & \frac{2 \pi f}{(2)^{1 / 2}} \cdot\left(\mu_{0} \cdot \varepsilon_{0}\right)^{1 / 2} \\
& \cdot\left\{\varepsilon_{r t}^{\prime} \cdot\left[\left(1+\frac{\varepsilon_{r t}^{\prime \prime 2}}{\varepsilon_{r t}^{\prime 2} \cdot \cos ^{2} \theta_{t}^{\prime}}\right)^{1 / 2}-1\right]\right\}^{1 / 2}
\end{aligned}
$$


with

$f=$ frequency $(4.5 \mathrm{GHz})$,

$\mu_{0}=$ magnetic permeability $\left(1.26 \times 10^{-6}\right)$,

$\varepsilon_{0}=$ air absolute dielectric permittivity

$$
\left(8.85 \times 10^{-12} \mathrm{Cv}^{-1} \mathrm{~m}^{-1}\right) \text {. }
$$

Thus, starting from the incident amplitude $E_{0}$, the transmitted amplitude $\left(E_{1}\right)$ from the soil surface to the top soil layer is given by

$$
E_{1}=E_{0} \cdot T_{0,1} \text {, }
$$

whereas the attenuated amplitude $\left(E_{1}^{\prime}\right)$ is given as follows:

$$
E_{1}^{\prime}=E_{1} \cdot \exp \left[-k_{1}^{\prime \prime}\left(z_{1}-z_{0}\right)\right] .
$$

Combining (10) and (11), we obtain

$$
E_{1}^{\prime}=E_{0} \cdot T_{0,1} \cdot \exp \left[-k_{1}^{\prime \prime}\left(z_{1}-z_{0}\right)\right] .
$$

For $n$ soil layers, the general form of Eq. (12) is given as follows:

$$
\begin{aligned}
E_{n}^{\prime}= & E_{0} \cdot \prod_{1, n}\left\{T_{i-1, i}\right\} \\
& \cdot \exp \left\{\sum_{1, n}\left[-k_{i}^{\prime \prime}\left(z_{i}-z_{i-1}\right)\right]\right\},
\end{aligned}
$$

where $z_{i}$ indicates the depth $i$.

Equation (13) provides an estimate for the "penetration depth" when the calculated limit of the ratio $\left(E_{n}^{\prime} / E_{0}\right)$ is equal to $(1 / e)$. For calculations, we used the estimated values for the real and imaginary parts of the permittivity given by Hallikainen et al. (1985), who used the soil texture and the volumetric water content as input. Calculations were made using an iterative procedure [Eq. (13)] after solving Eqs. (4)-(7), and thus calculating the transmission and attenua- tion coefficients [Eqs. (8) and (9)] for each soil layer.

\section{Results}

\section{Empirical results from water content and water potential data}

Figure 2 shows the 17 average experimental volumetric water content profiles including wet, intermediate, and dry soil conditions. The near-surface water gradients are sometimes high, particularly for dry conditions. Standard deviations relative to the volumetric water contents were generally between 0.010 and 0.050 $\mathrm{cm}^{3} \cdot \mathrm{cm}^{-3}$. As indicated before, a preliminary geostatistical analysis was performed to insure a statistical independence between replicates for each day. Results showed no spatial correlations between measurements for distances between sampling locations greater than $4 \mathrm{~m}$.

Figures 3 and 4 show the experimental relationships between the backscattering coefficient and, respectively, volumetric water content $(0-5 \mathrm{~cm})$ and water potential for two incidence angles. The regression line between the backscattering coefficient and the water potential (log scale) is obtained over a wide range of wet or dry conditions: These results are in agreement with those that Dobson and Ulaby (1981) provided for a limited soil tension range.

Similar regression lines between the backscattering coefficient and volumetric water content were calculated for different arbitrary soil layer depths. Table I shows how increasing the sampling depth affects the mean moisture for a given gradient or profile. Although the volumetric water content profiles presented high gradients, correlation coefficients were between 0.859 and 0.899 for the 
volumetric water content

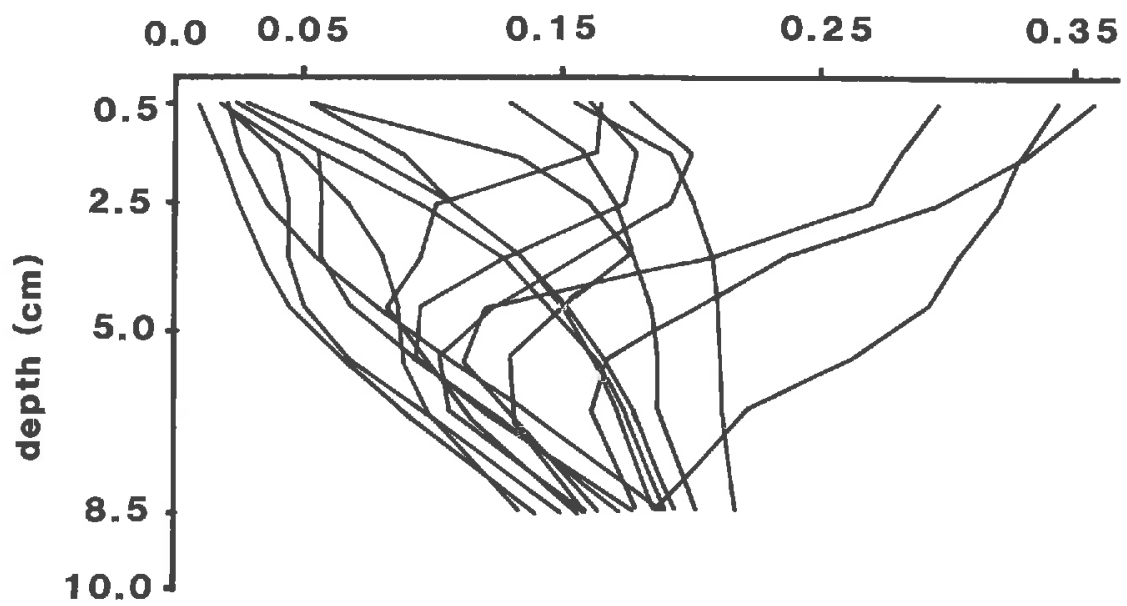

FIGURE 2. Mean experimental volumetric water content profiles from 0 to $10 \mathrm{~cm}$ $\left(\mathrm{cm}^{3} \cdot \mathrm{cm}^{-3}\right)$.

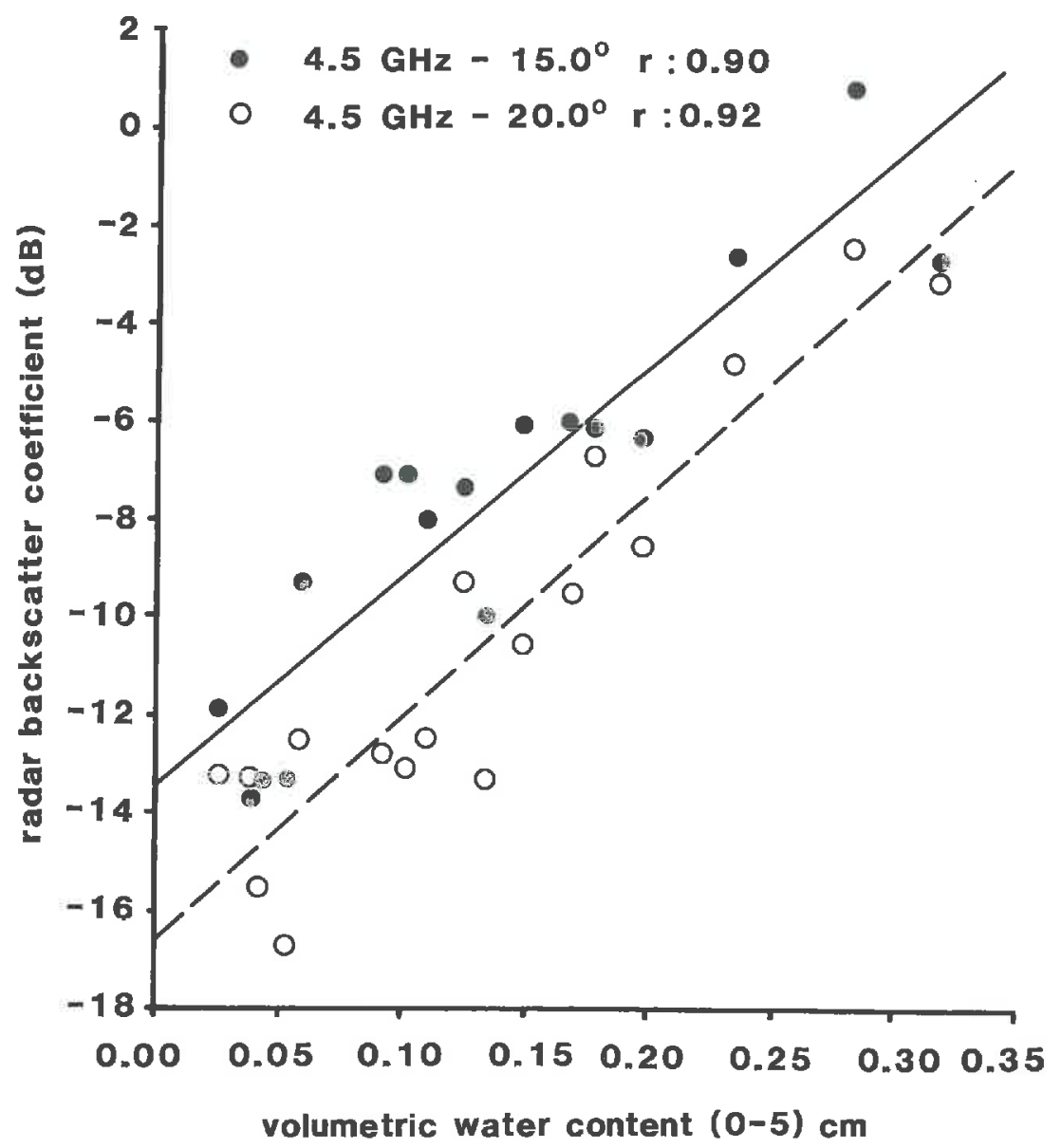

FIGURE 3. Experimental relationship between backscattering coefficient and the volumetric water content $(0-5 \mathrm{~cm})$ for two incidence angles $\left(15^{\circ}\right.$ and $\left.20^{\circ}\right)$. 


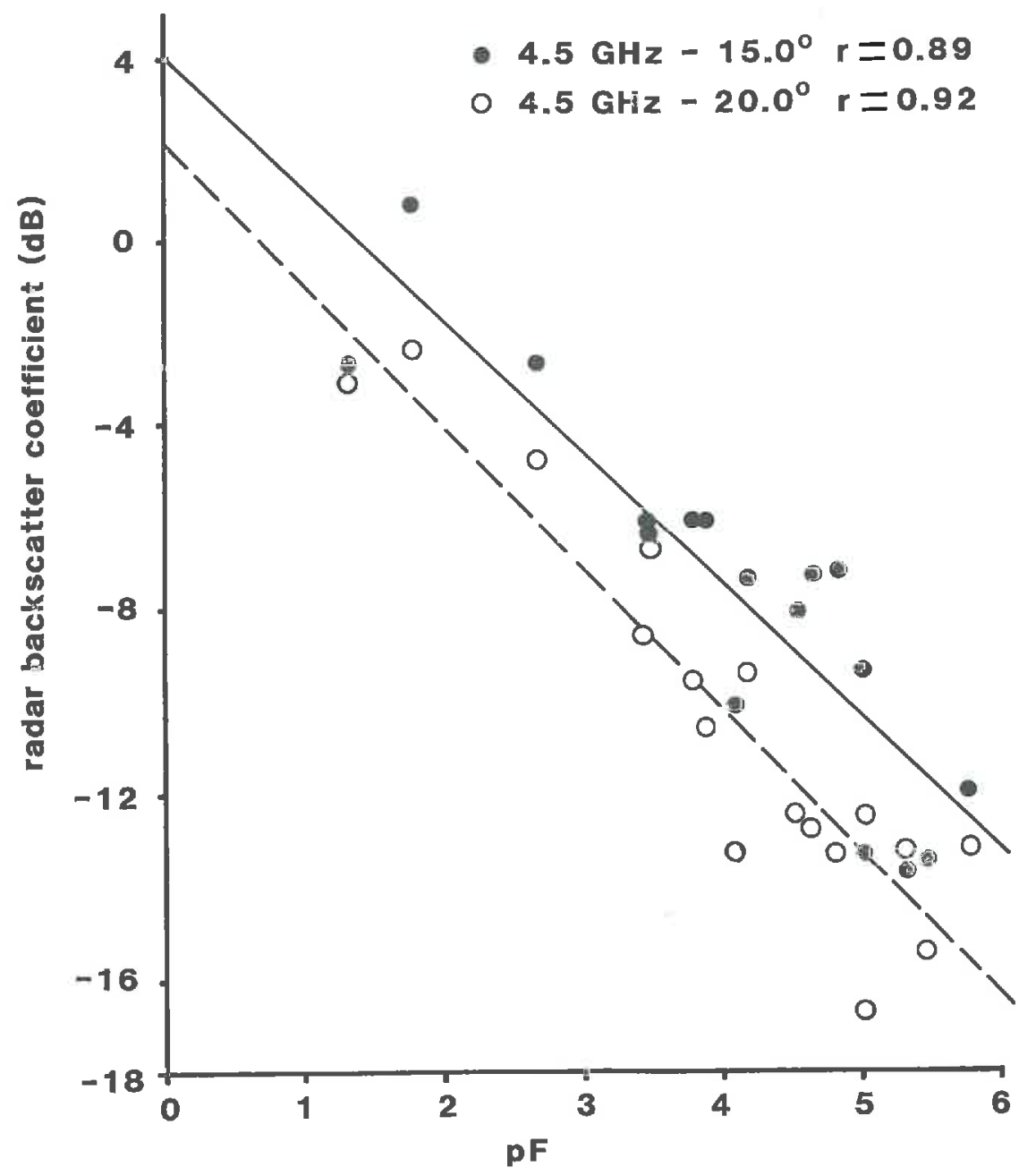

FIGURE 4. Experimental relationship between the backscattering coefficient and the water potential $(0-5 \mathrm{~cm}, \log \mathrm{scale})$ for two incidence angles $\left(15^{\circ}\right.$ and $\left.20^{\circ}\right)$.

$4.5 \mathrm{GHz}$ frequency and $15^{\circ}$ look angle (Table 2). Figure 5 shows the calculated residuals after regression: The highest residuals after regression analysis are obtained for small ( $0-1 \mathrm{~cm}$, for example) or great $(0-10 \mathrm{~cm}$, for example) soil depths, whereas intermediate soil layers $(0-5 \mathrm{~cm}$, for example) exhibit the smallest residuals. Furthermore, a particular trend of variation of the residuals vs. the mean volumetric water content may be visible.

With small soil depths, the residuals are high for dry soil conditions. With great soil depths, they seem high for wet soil conditions. Data analysis showed that this was not explained by the effect of the variance of the ground measurements on the residuals, particularly for the low water contents that had a small standard deviation $\left(0.01-0.02 \mathrm{~cm}^{3} / \mathrm{cm}^{3}\right)$. This is probably related to soil thickness which determines the microwave penetration depth: Dry soil conditions generally correspond to great soil penetration depths, and, thus, taking into account a small soil depth may induce nonnegligible errors. 
TABLE 1 Experimental Volumetric Water Content when Different Soil Layers are taken into Account $(0-1$ to $0-10 \mathrm{~cm})$

\begin{tabular}{rcccccccc}
\hline $\begin{array}{c}\text { Profile } \\
\text { No. }\end{array}$ & $0-1$ & $0-2$ & $0-3$ & $0-4$ & $0-5$ & $0-6$ & $0-7$ & $0-10$ \\
\cline { 2 - 8 } 1 & 0.020 & 0.023 & 0.027 & 0.034 & 0.043 & 0.054 & 0.066 & 0.103 \\
2 & 0.015 & 0.032 & 0.044 & 0.053 & 0.059 & 0.064 & 0.069 & 0.089 \\
3 & 0.025 & 0.041 & 0.046 & 0.049 & 0.053 & 0.060 & 0.068 & 0.100 \\
4 & 0.161 & 0.171 & 0.172 & 0.162 & 0.148 & 0.139 & 0.136 & 0.149 \\
5 & 0.154 & 0.173 & 0.182 & 0.189 & 0.193 & 0.196 & 0.198 & 0.204 \\
6 & 0.128 & 0.144 & 0.153 & 0.159 & 0.164 & 0.168 & 0.171 & 0.180 \\
7 & 0.051 & 0.070 & 0.083 & 0.096 & 0.107 & 0.117 & 0.125 & 0.146 \\
8 & 0.026 & 0.050 & 0.069 & 0.086 & 0.099 & 0.109 & 0.118 & 0.140 \\
9 & 0.022 & 0.040 & 0.058 & 0.075 & 0.089 & 0.101 & 0.111 & 0.134 \\
10 & 0.009 & 0.013 & 0.016 & 0.020 & 0.025 & 0.032 & 0.040 & 0.070 \\
11 & 0.017 & 0.029 & 0.034 & 0.036 & 0.039 & 0.044 & 0.051 & 0.081 \\
12 & 0.177 & 0.189 & 0.190 & 0.183 & 0.170 & 0.159 & 0.152 & 0.154 \\
13 & 0.165 & 0.165 & 0.147 & 0.134 & 0.124 & 0.119 & 0.118 & 0.129 \\
14 & 0.294 & 0.287 & 0.281 & 0.263 & 0.234 & 0.214 & 0.201 & 0.190 \\
15 & 0.054 & 0.094 & 0.116 & 0.132 & 0.135 & 0.134 & 0.134 & 0.141 \\
16 & 0.340 & 0.333 & 0.328 & 0.321 & 0.315 & 0.306 & 0.294 & 0.261 \\
17 & 0.354 & 0.342 & 0.322 & 0.301 & 0.282 & 0.263 & 0.248 & 0.228 \\
\hline
\end{tabular}

TABLE 2 Statistical Characteristics of the Experimental Regression Lines between the Backscattering Coefficient and the Mean Volumetric Water Content for Arbitrary Soil Depths (4.5 $\mathrm{GHz}, \mathrm{HH}$ polarization, $15^{\circ}$ look angle).

\begin{tabular}{cccc}
\hline $\begin{array}{c}\text { DePtH } \\
(\mathrm{cm})\end{array}$ & SLOPE & INTERCEPT & $\begin{array}{c}\text { CORRELATION } \\
\text { CoEFFicIENT }\end{array}$ \\
\hline $0-1$ & 29.8 & -11.2 & 0.865 \\
$0-2$ & 32.6 & -11.9 & 0.878 \\
$0-3$ & 35.5 & -12.5 & 0.892 \\
$0-4$ & 38.9 & -13.0 & 0.899 \\
$0-5$ & 42.6 & -13.4 & 0.897 \\
$0-6$ & 46.5 & -13.9 & 0.889 \\
$0-7$ & 50.8 & -14.6 & 0.880 \\
$0-10$ & 66.1 & -17.4 & 0.859 \\
\hline
\end{tabular}

Wet soil conditions generally correspond to very small microwave penetration depths, and, thus, taking into account a high soil depth is probably unsatisfactory if large gradient exists. Thus, the residuals may increase when these phenomena are unadequately taken into account.

Finally, according to a classical calibration procedure, the data analysis provides statistical empirical regression lines that exhibit a quite strong dependence of backscattering coefficient on either water content or water potential, and the residuals analysis is probably explained in terms of microwave penetration depth effects.

\section{Microwave penetration depth: theoretical and experimental results}

In the classical calibration procedure, analysis of regression lines is performed using arbitrary soil depths, although water content gradients near the soil surface are sometimes high. Thus, it would be rea- 

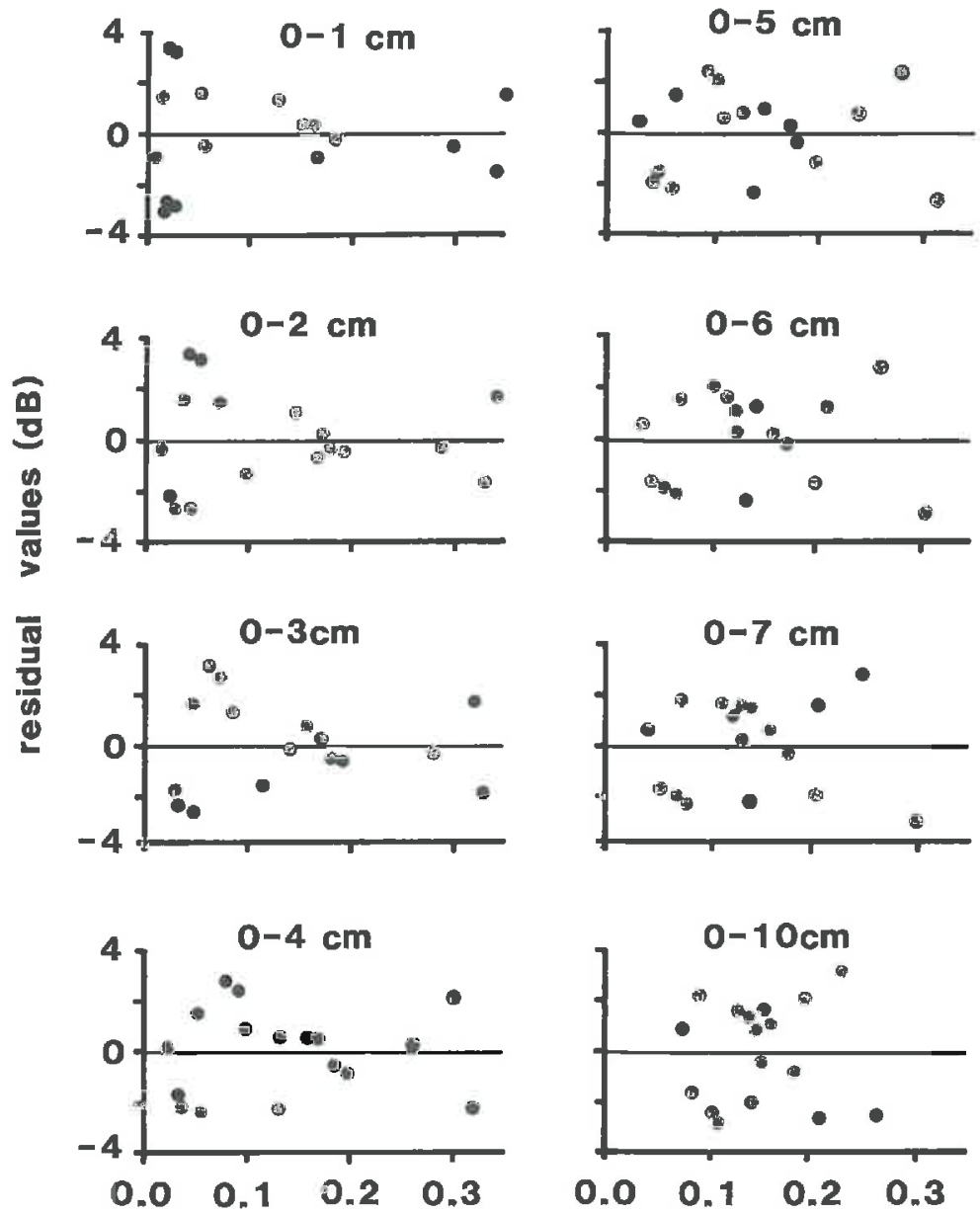

\section{volumetric water content}

FIGURE 5. Residual values for the backscattering coefficients after regression analysis using different soil depths $\left(4.5 \mathrm{GHz}, \mathrm{HH}\right.$ polarization, $\left.15^{\circ}\right)$.

sonable to take into account the depth whose moisture content determines the soil surface reflectivity to obtain a more precise and physically based regression curve between backscattering coefficient and water content. According to a physical point of view, the "soil moisture sampling depth" is the depth whose moisture content determines the surface reflectivity and emissivity. While the "microwave penetration depth" is the depth at which the field strength has decreased to $1 / e$.
Both theoretical (Wilheit, 1978) and experimental studies (Newton et al., 1983) have shown that the thickness of the layer at the surface which determines the surface reflectivity is on the order of a few tenths of a wavelength, i.e., about 1 $\mathrm{cm}$ at C-band. According to a practical point of view, the use of the "soil moisture sampling depth" is quite difficult to achieve because it is rather difficult to measure accurately soil water content for thin soil layers $(\leqslant 1 \mathrm{~cm})$. 
The microwave penetration depth is greater than the soil moisture sampling depth and thus more compatible with the experimental field conditions of water content measurements. An example is presented on Fig. 6. Calculations took into account two cases: i) Soil water content was assumed to be uniform, and the theoretical relationship between the penetration depth and the volumetric water content was established (solid line on Fig. 6); ii) the same calculations were made but using the 17 experimental and obviously nonuniform water content profiles (closed circles on Fig. 6). Results show that the microwave penetration depth is approximately between 0.0 and $10.0 \mathrm{~cm}$ for a large range of experimental water content profiles having different shapes and that nonuniform shape of the volumetric water content profiles induces only a small difference compared with the microwave penetration depth relationship when unifoim water content profiles are assumed. This appears clearly with the following theoretical example: Four pairs of water content profiles were chosen over a range of volumetric water contents between 0.10 and 0.35 . For each pair, the mean water content was exactly

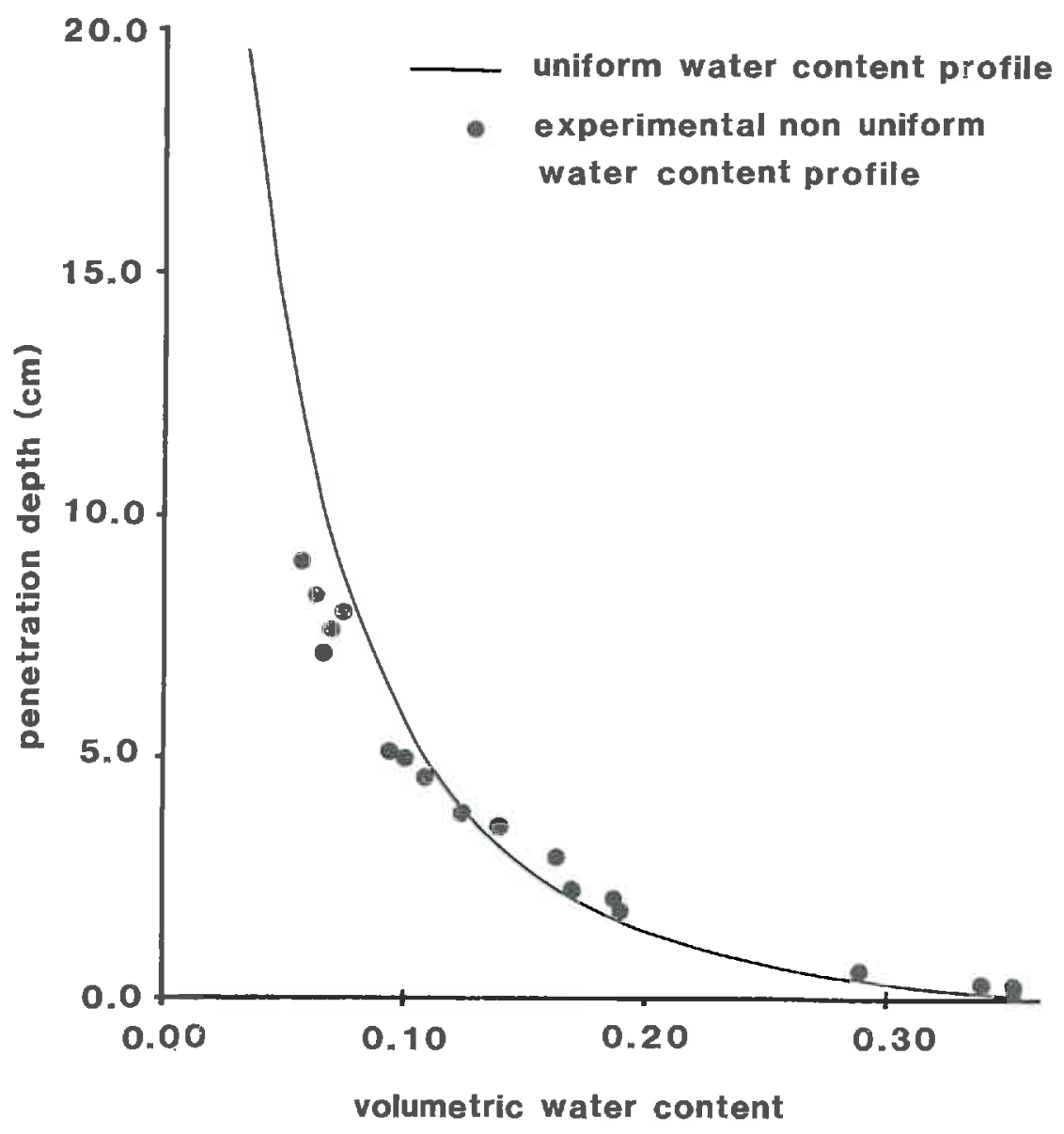

FIGURE 6. "Microwave penetration deptl/volumetric water content" relationship for uniform and experimental nomuniform water content profiles (4.5 $\mathrm{GHz}, \mathrm{HH}$ polarization, $15^{\circ}$ ). 
volumetric water content
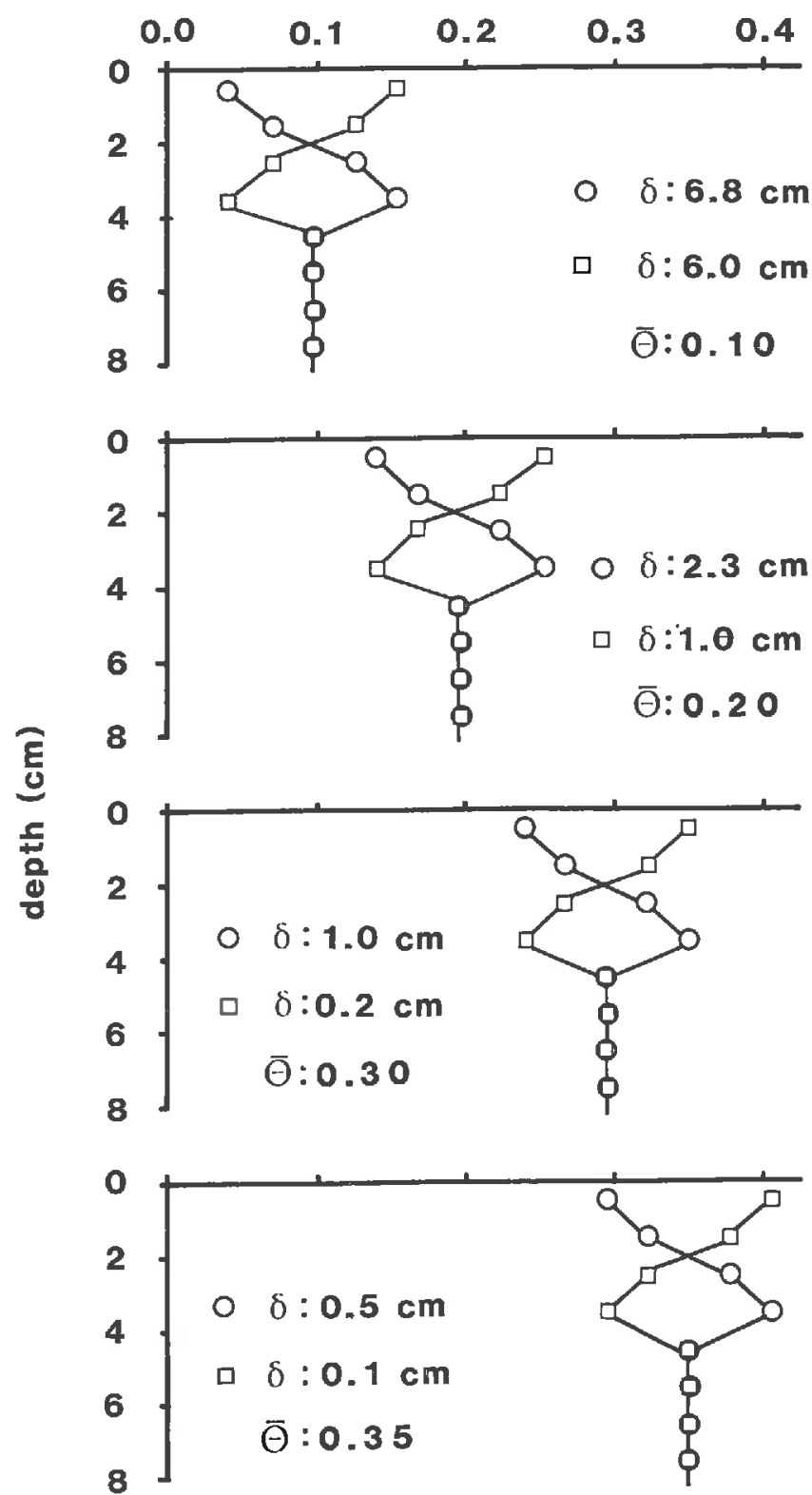

FIGURE 7. Calculated microwave penetration depths for contrasted theoretical water content profiles. ( $\delta$ is the calculated microwave penetration clepth; $4.5 \mathrm{GHz}, \mathrm{HH}$ polarization, $\left.15^{\circ}\right)$. 
the same, but the shape of the two profiles was symmetric from the soil surface to the $5 \mathrm{~cm}$ depth (Fig. 7). As indicated on Fig. 7, results show that i) the main effect on the calculated penetration depth is due to the mean water content and ii) a secondary effect is dependent on the actual shape of the water content profile.

Consequently, the microwave penetration depth is a sensitive factor to the actual shape of any water content profile and the order of magnitude of the calculated microwave penetration depth seems compatible with the thickness of soil layers that are taken into account during the soil water content measurements. We will analyze here if the microwave penetration depth is of interest in order to obtain more accurate regression curves between the backscattering coefficient and soil volumetric water content.

Table 3 shows the differences between the mean and volumetric water contents calculated for an arbitrary soil depth (0-5 $\mathrm{cm}$ ) and from the soil surface to the calculated penetration depth for the 17 profiles. Systematic differences appear for wet $(\delta<5 \mathrm{~cm})$ and dry $(\delta>5 \mathrm{~cm})$ soils, which are explained both by the mean volumetric water content and profile shapes as indicated above. From a statistical point of view, Table 4 compares the
TABLE 3 Comparison between the Volumetric Water Content Calculated over an Arbitrary Depth (0-5 cm) and the Volumetric Water Content Calculated from the Soil Surface to the Microwave Penetration Depth $(0-\delta \mathrm{cm})$.

\begin{tabular}{ccc}
\hline$\Theta_{0-5}$ & $\Theta_{0-\delta}$ & $\left(\Theta_{0-5}-\Theta_{0-\delta}\right)$ \\
\hline 0.025 & 0.057 & -0.032 \\
0.039 & 0.064 & -0.025 \\
0.043 & 0.068 & -0.025 \\
0.053 & 0.071 & -0.018 \\
0.059 & 0.074 & -0.015 \\
0.091 & 0.095 & -0.004 \\
0.101 & 0.101 & 0.000 \\
0.109 & 0.108 & 0.001 \\
0.123 & 0.140 & -0.017 \\
0.134 & 0.124 & 0.010 \\
0.148 & 0.169 & -0.021 \\
0.168 & 0.165 & 0.003 \\
0.176 & 0.190 & -0.014 \\
0.197 & 0.189 & 0.008 \\
0.234 & 0.294 & -0.060 \\
0.281 & 0.354 & -0.073 \\
0.316 & 0.342 & -0.026 \\
\hline
\end{tabular}

calculated first- or second-order regression curves with or without taking into account the microwave penetration depth. Results show that the hypothesis of a second-order relationship between the backscattering coefficient and the volumetric water content is statistically better than the first-order relationship assumption only when the microwave penetration depths are taken into account, whereas no changes between the two regression curves are observed when

TABLE 4 Statistical Comparison between First- and Second-Order Regression Curves with or without Taking into Account the Microwave Penetration Depth $(4.5 \mathrm{GHz}, \mathrm{HH}$ polarization, $15^{\circ}$ look angle)

\begin{tabular}{cllcc}
\hline $\begin{array}{c}\text { INCIDENCE } \\
\text { ANGLE }\end{array}$ & \multicolumn{2}{c}{$\begin{array}{c}\text { Regression } \\
\text { CuRve }\end{array}$} & $\Theta(0-5 \mathrm{~cm})$ & $\Theta(0-8 \mathrm{~cm})$ \\
\hline $15^{\circ}$ & first order & $r^{2}$ & 0.805 & 0.814 \\
& & residual & 0.038 & 0.042 \\
& \multirow{2}{*}{ second order } & $r^{2}$ & 0.826 & 0.884 \\
& & residual & 0.038 & 0.035 \\
$20^{\circ}$ & first order & $r^{2}$ & 0.863 & 0.906 \\
& & residual & 0.033 & 0.030 \\
& \multirow{3}{*}{ second order } & $r^{2}$ & 0.872 & 0.951 \\
& & residual & 0.033 & 0.023 \\
\hline
\end{tabular}


penetration depth effects are neglected. A detailed example is provided on Fig. 8 for the $4.5 \mathrm{GHz}, \mathrm{HH}$ polarization, and $15^{\circ}$ look angle configuration. These results agree with the theoretical calculations which showed that the "reflection coefficient/water content" relationship is nonlinear (Pausader, 1982).

Estimating soil water contents

from microwave measurements

For practical applications, researchers are interested in a more or less accurate estimation of near-surface soil water content: The classical (see Fig. 3) or mod- ified (see Fig. 8) regression curve analysis makes it possible to estimate the mean water content over a given soil depth. The quality of the estimates depends on the quality of the estimated regression curve, and no precise information on the water content at the soil surface is provided. Furthermore, when the researchers are interested in the use of near-surface water content estimates as boundary conditions in "soil/atmosphere" water flows models, a precise estimated water content just at the soil surface is what is sought: As a matter of fact, "soil/atmosphere" water flows models have generally a great sensitivity to errors in the boundary con-

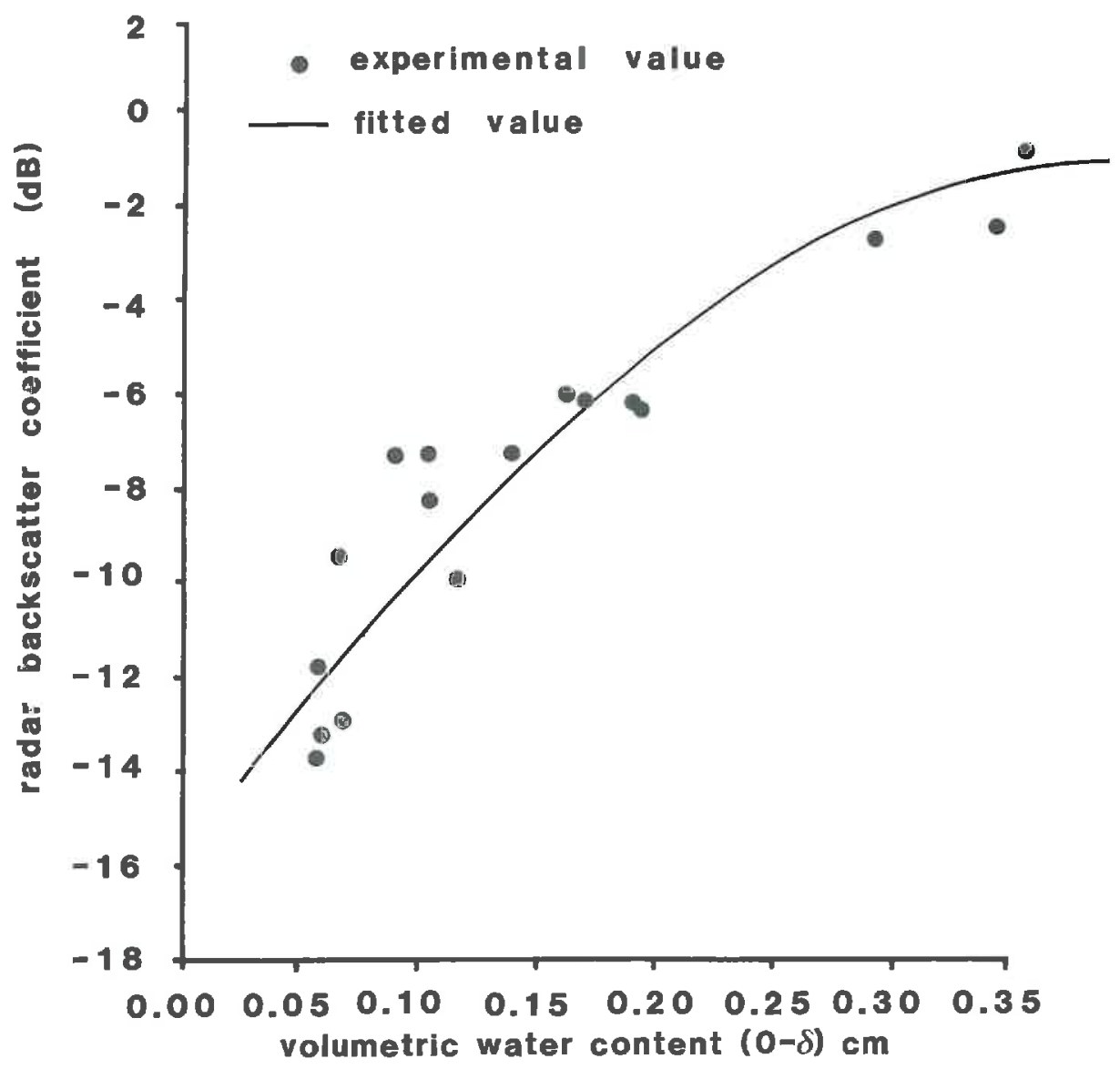

FIGURE 8. Experimental nonlinear relationship between the backscattering coefficient and the volumetric water content from the soil surface to the microwave penetration depth $(0-\delta \mathrm{cm})$ for the $4.5 \mathrm{GHz}, \mathrm{HH}$ polarization, and $15^{\circ}$ look angle. 
ditions. Thus, it is important to improve, if possible, the soil water content estimates at the soil surface from microwave data in order to minimize the propagation of errors when accurate "soil/ atmosphere" water transfers models are used. In order to obtain such information on the near-surface water content, we suggest testing the following mathematical procedure.

\section{Basic equations}

We assume that we have different empirical calibration lines between the backscattering coefficient and volumetric water content for several arbitrary soils depths $(0-1,0-2, \ldots, 0-10 \mathrm{~cm}$, for example). In the following equations, the subscript 1 refers to the first regression line, and the subscript $n$ to the last one. The symbols $a$ and $b$ correspond to the slope and intercept of the regression lines, respectively, whereas the notations $\Theta_{i, i+1}$ and $\sigma_{0}$ correspond to the mean volumetric water content between the depths $i$ and $i+1$ and the backscattering coefficient, respectively. Thus, we obtain

$$
\begin{gathered}
a_{1} \cdot \Theta_{0,1}+b_{1}=\sigma_{0}, \\
a_{2} \cdot \Theta_{0,2}+b_{2}=\sigma_{0}, \\
\ldots \\
a_{n} \cdot \Theta_{0, n}+b_{n}=\sigma_{0} .
\end{gathered}
$$

Consider now that we have an obvious set of relationships between the $\left(\Theta_{0,1}\right.$, $\left.\Theta_{1,2}, \ldots, \Theta_{i-1, i}, \ldots, \Theta_{n-1, n}\right)$ :

$$
\begin{aligned}
& \Theta_{0,1}=\alpha_{11} \cdot \Theta_{0,1}, \\
& \Theta_{0,2}=\alpha_{21} \cdot \Theta_{0,1}+\alpha_{22} \cdot \Theta_{1,2}, \\
& \cdots \\
& \Theta_{0, n}=\alpha_{n 1} \cdot \Theta_{0,1}+\cdots+\alpha_{n n} \cdot \Theta_{n-1, n},
\end{aligned}
$$

where the $\left(\alpha_{i j}\right)$ coefficients are dimensionless weighting factors that account for the elementary thickness of the soil layers chosen during the water content sampling procedure. Introducing (15) in (14) and recombining, we obtain in a matrix form

$$
[a \cdot \alpha] \cdot\{\Theta\}=\left\{\sigma_{0}-b\right\},
$$

which is a linear set of equations to be solved in terms of $\{\Theta\}$ :

$$
\begin{gathered}
a_{1} \cdot \alpha_{11}, \quad\left\{\Theta_{0,1}\right\}=\sigma_{0}-b_{1} \\
a_{2} \cdot \alpha_{21}+a_{2} \cdot \alpha_{22}, \quad\left\{\Theta_{1,2}\right\}=\sigma_{0}-b_{2} \\
\cdots \\
a_{n} \cdot \alpha_{n 1}+\cdots+a_{n} \cdot \alpha_{n n}, \\
\quad\left\{\Theta_{n-1, n}\right\}=\sigma_{0}-b_{n}
\end{gathered}
$$

Thus, calculations using Eq. (17) provide an estimation of the entire water content profile from the layer 1 to the layer $n$, and a procedure of extrapolation until the soil surface $(z=0)$ may easily provide the estimated water content just at the soil surface. Note that for the water content profile estimation, only one measurement of backscattering coefficient $\left(\sigma_{0}\right)$, but several calibration lines $\left(a_{i}, b_{i}\right)$ are necessary, one for each arbitrary soil layer. If the last soil layer $n$ is chosen as corresponding to the microwave penetration depth, the set of linear equations (17) will provide an estimated water con- 
tent profile from the first layer to the microwave penetration depth.

\section{Statistical point of view}

Different terms appear in Eq. (17):

i. Weighting dimensionless factors $\left(\alpha_{i j}\right)$.

ii. Slopes and intercepts of the different regression lines $\left(a_{i}, b_{i}\right)$.

iii. Measured backscattering coefficient $\left(\sigma_{0}\right)$.

iv. Calculated water content between the depths $i$ and $i+1,\left(\Theta_{i, i+1}\right)$.

The terms $\mathrm{i}$-iii are used as input, whereas the terms iv are what is sought. According to a statistical point of view, the terms i are considered as constant, whereas the terms ii and iii are regarded as random variables, because some uncertainties due to measurement errors and spatial variability appear during the calibration procedure (ii) and backscattering measurements (iii). Subsequently, the calculated results (iv) are also considered as random variables. Thus, the variance of the slope and intercept of the regression lines correspond to errors due to the calibration procedure, whereas the variance of the backscattering coefficient is involved in any measurement (calibration phase or not). To take into account the errors coming from the calibration procedure, the analysis was conducted as follows:

The statistical distribution of the backscattering coefficient measured during the calibration phase, and of the measured water content as well, were assumed to be normal. The estimated standard deviation (SD) for the backscattering coefficient was taken as equal to $0.5 \mathrm{~dB}$ and the standard deviation for the volumetric water content was chosen in agreement with the measured spatial variability (generally between 0.01 and 0.05 $\left.\mathrm{cm}^{3} / \mathrm{cm}^{3}\right)$. Starting from the mean values obtained during the experiment (17 days), 100 independent values for each of the 17 backscattering coefficients and 17 volumetric water contents were extracted at random according to their normal probability law, respectively. Thus, it was possible to simulate 100 calibration lines, and subsequently 100 slopes and intercepts. This was done for each arbitrary soil layer $(0-1$ to $0-10 \mathrm{~cm})$ in order to take into account the variability of each calibration line corresponding to each different soil layer. Results are given in Figs. 9a) and 9b). They show that the estimated slopes and intercepts for each calibration line exhibit a nonnegligible sensitivity to uncertainties in the input data used during the calibration phase (backscattering coefficient and volumetric water content).

\section{Checking the estimation procedure}

Calculations were made using Eq. (17) and the previous statistical results to compare the predicted and measured values $(\Theta)$ for 15 experimental water content profiles (two sets of data were eliminated because they had exactly the same measured backscattering coefficient as two others and would provide the same results). Mean values and standard deviations (SD) were calculated for the estimated volumetric water content, respectively. The standard deviations (SD) were calculated using an iterative procedure as follows: For each of the 15 measured values of the backscattering coefficient, i) 30 values were generated at random in agreement with the normal probability law to simulate the measurement errors during the experiment and ii) for each 


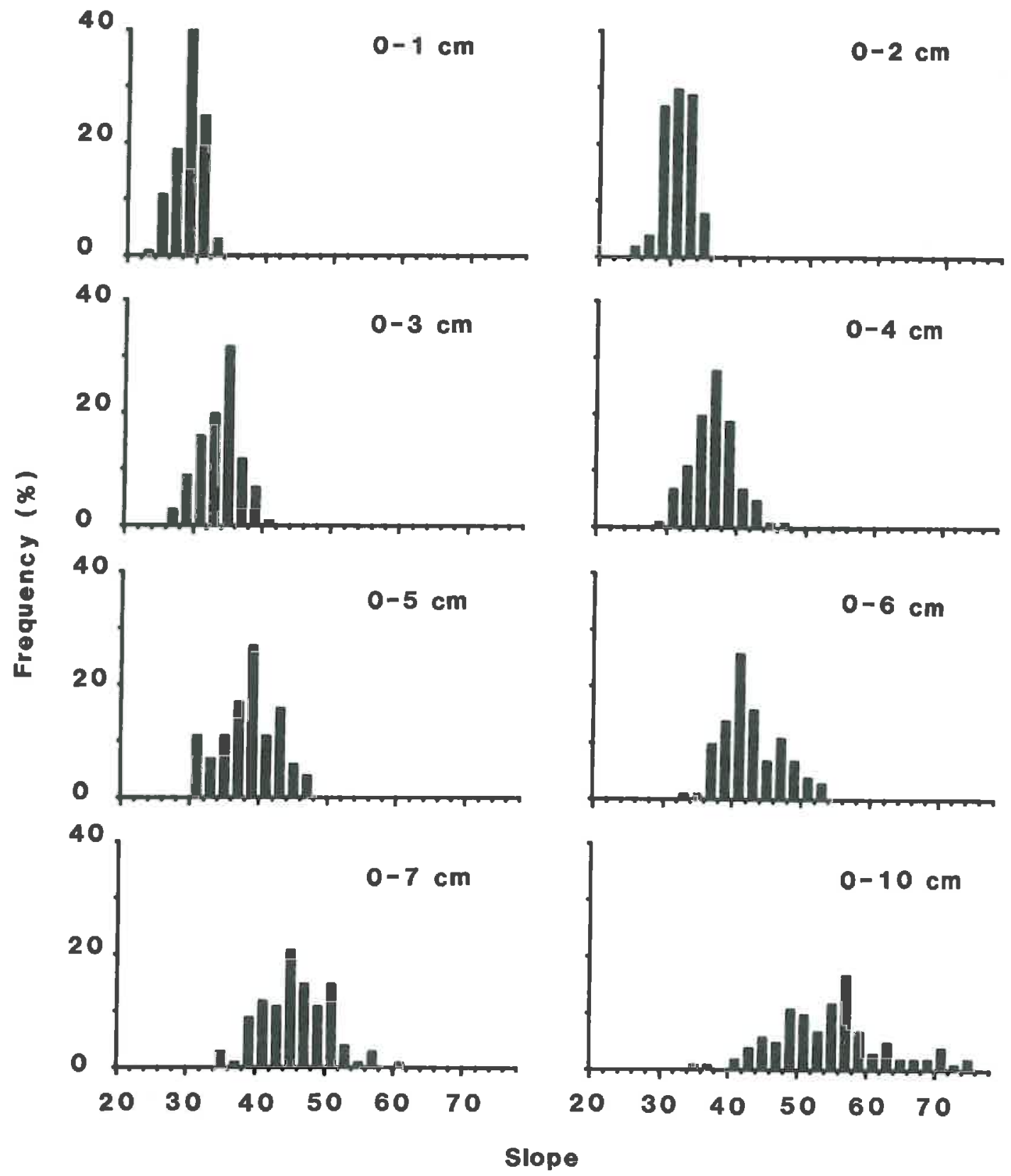

FIGURE 9(a). Frequency distribution of the slopes of the "backscattering coefficient/volumetric vater content" regression lines when measurement errors are taken into account $(0-1 \mathrm{~cm}$ to $0-10 \mathrm{~cm})$. 


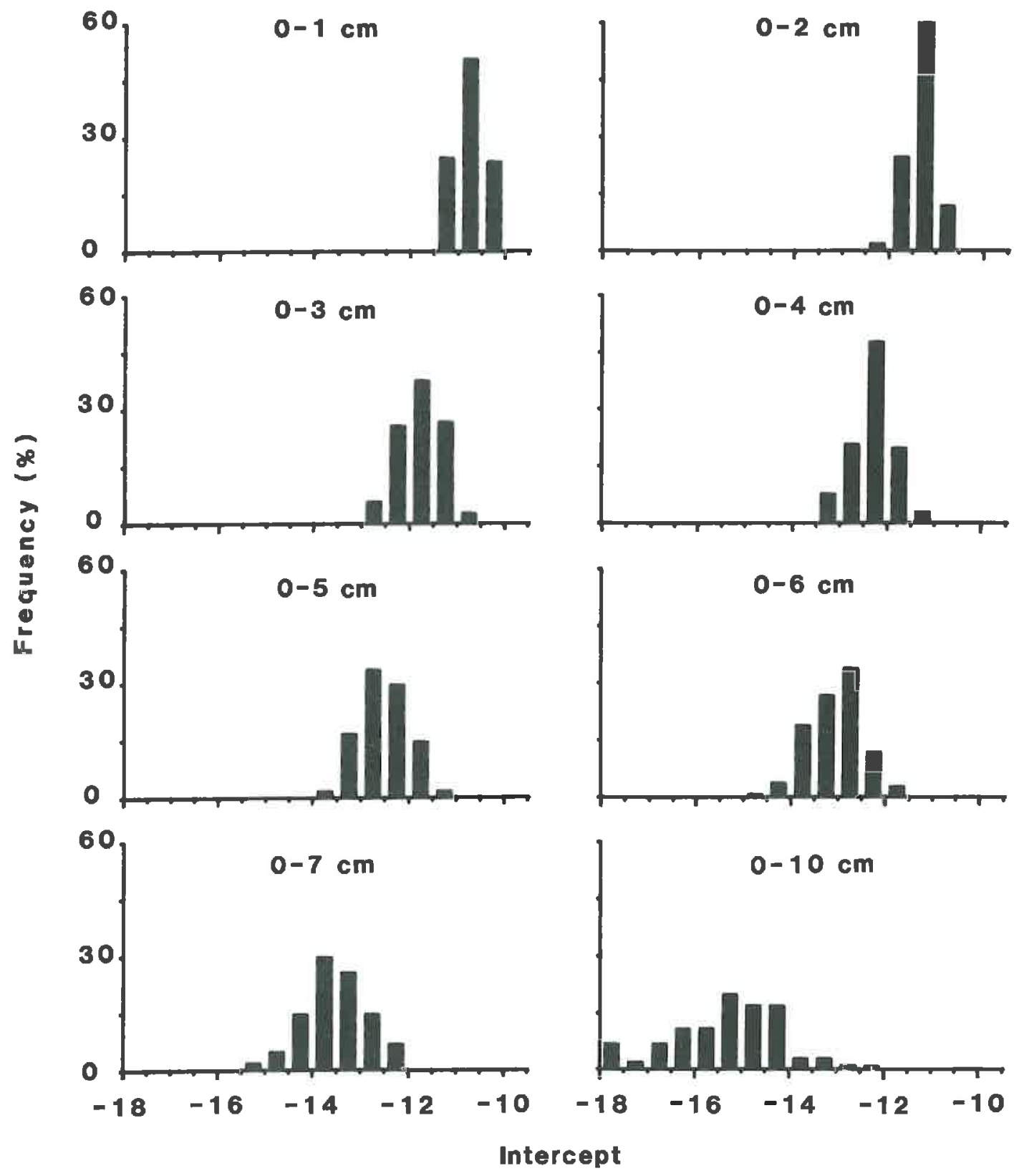

FIGURE 9(b). Frequency distribution of the intercepts of the "backscattering coefficient/volumetric water content" regression lines when measurement errors are taken into account $(0-1 \mathrm{~cm}$ to $0-10 \mathrm{~cm})$. 
generated backscattering coefficient, a set of calibration lines was selected according to their probability distribution as given in Figs. 9a) and 9b).

Figure 10 shows the statistical relationship between the observed and calculated mean volumetric water contents and accounts only for soil layers between the soil surface and the calculated microwave penetration depth: The general trend is satisfactory, although some differences may be greater than or equal to 0.05 $\mathrm{cm}^{3} / \mathrm{cm}^{3}$. As a matter of fact, note that the observed and calculated mean volumetric water contents on Fig. 10 correspond to different soil layers located between depths $i$ and $i+1$, and that the precision of the estimates for individual layers between depths $i$ and $i+1$ is of the same order of magnitude as the precision for mean water content estimates
(0-5 cm, for example) with a classical calibration procedure (see Fig. 3).

Figure 11 gives the detailed comparison for each observed and estimated water content profile from the soil surface to the calculated penetration depth. Nearsurface water contents and profile shapes are quite well calculated especially for dry and wet soils. For the intermediate water contents obtained using short irrigation over a dry soil surface, water content profiles were irregular (see Fig. 2), and, thus, the corresponding points used for the calibration line analysis taking into account an arbitrary soil depth are in fact difficult to interpret. From a statistical point of view, the calculated standard deviations are generally quite important, particularly at the bottom of the water content profile. This is due partly to the frequency distribution of the slopes and

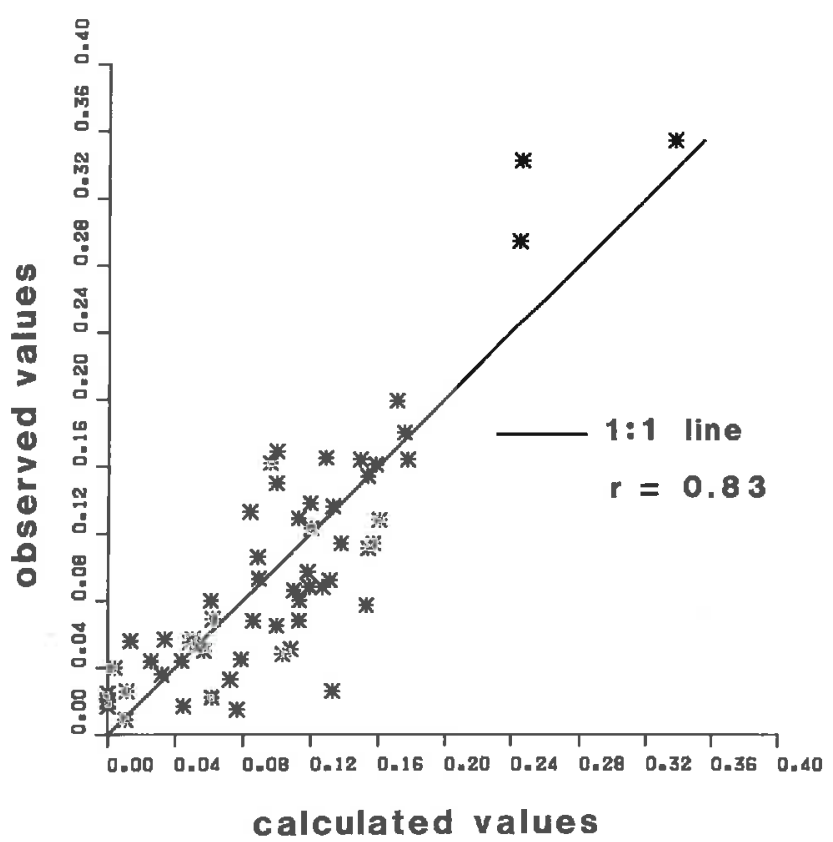

FIGURE 10. Measured and predicted mean volumetric water content for different soil layers between 0 and the microwave penetration depth $\delta$ [Eq. (17)] $\left(4.5 \mathrm{GHz}, \mathrm{HH}\right.$ polarization, $\left.15^{\circ}\right)$. 
Volumetric water content
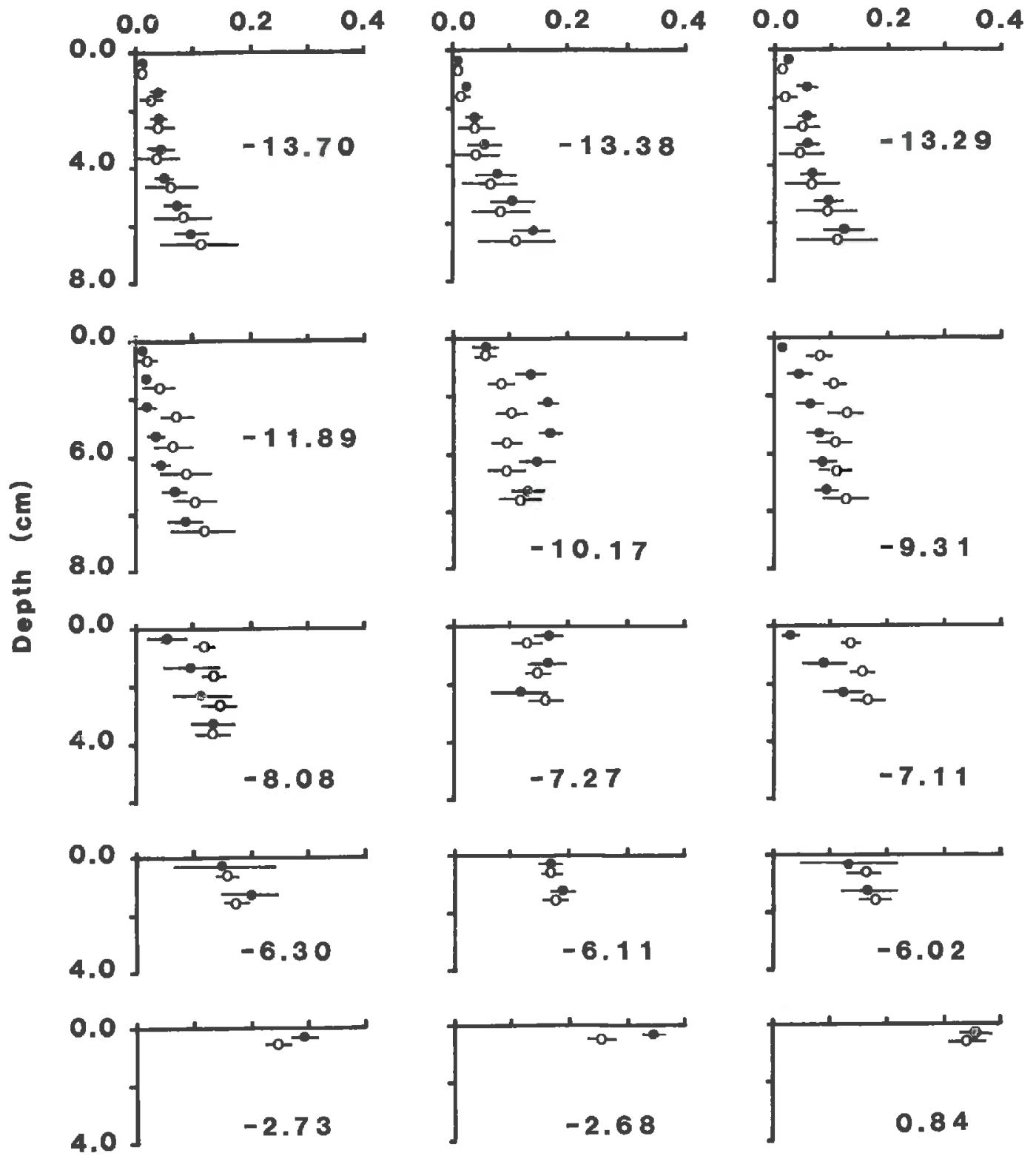

FIGURE 11. Detailed comparison between measured and predicted water content profiles [Eq. (17)]; numerical values indicate the measured backscattering coefficient $(\mathrm{dB}) ;\left(4.5 \mathrm{GHz}, \mathrm{HH}\right.$ polarization, $\left.15^{\circ}\right):(\oplus)$ observed value $\pm \mathrm{SD}$; $(\theta)$ calculated value $\pm \mathrm{SD}$. 
intercepts of the regression lines [see Figs. $9 a)$ and $9 b$ )], which become greater when the thickness of the sampling depth increases.

\section{Conclusion}

Precise information on near-surface water content or water potential is of interest for several applications, particularly for the modeling of water movements from the soil surface to the atmosphere.

In order to obtain such information, theoretical and empirical results were presented to test the capability of the backscattering coefficient to provide precise information about the near-surface soil water content. Results indicated that the mean water content and secondly the profile shape had an important effect on the estimated microwave penetration depth, and that the proposed approach for estimating soil water content profiles was encouraging. However, the proposed approach will provide satisfactory estimates only if the volumetric water content profile to be estimated is in the same range as those used during the calibration procedure. Thus, starting with a uniform wet water content profile and then drying under natural conditions, we will obtain empirical regression lines between the backscattering coefficient and the volumetric water content, which will be able to give satisfactory estimates of any water content profile corresponding to the same range of climatic conditions.

Further investigations are to be performed that will test the presented procedure under various field conditions, and use such predicted soil surface water contents as boundary conditions for model- ing soil water flow, using empirical or deterministic models.

\section{References}

Becker, F. (1978). Fundamental physics of remote sensing, in Mathematical and Physical Principles of Remote Sensing, 1-107, Summer School of Space Physics, Strasbourg (FRA), 18 August-15 September 1978, Centre National d'Etudes Spatiales, France.

Bernard, R., Vauclin, M., and Vidal-Madjar, D. (1981), Possible use of active microwave remote sensing data for prediction of regional evaporation by numerical simulation of soil water movement in the unsaturated zone, Water Resour. Res. 17(6):1603-1610.

Bernard, R., Martin, Ph., Thony, J. L., Vauclin, M., and Vidal-Madjar, D. (1982), C-Band radar for determining surface soil moisture, Remote Sens. Environ. 12:189-200.

Bernard, R., Soares, J. V., and Vidal-Madjar, D. (1986), Differential bare field drainage properties from airborne microwave observations, Water Resour. Res. 22:869-875.

Bertuzzi, P., Bruckler, L., Gabilly, Y., and Gaudu, J. C. (1987), Calibration, field testing and error analysis of a gamma-ray probe for the in situ measurement of dry bulk density, Soil Sci. 144(6):425-436.

Bradley, G. A., and Ulaby, F. T. (1981), Aircraft radar response to soil moisture, Remote Sens. Environ. 11:419-438.

Dobson, M. C., and Ulaby, F. T. (1981), Microwave backscatter dependence on surface roughness, soil moisture, and soil texture: Part III-Soil tension, IEEE Trans. Geosci. Remote Sens. GE-19(1):51-61.

Hallikainen, M. T., Ulaby, F. T., Dobson, M. C., El-Rayes, M. A., and $\mathbf{W u}, \mathbf{L}$. K. (1985), Microwave dielectric behaviour of wet soil. Part I: Empirical models and ex- 
perimental observations, IEEE Trans. Geosci. Remote Sens. 23(1):25-34.

Hirosawa, H., Komiyama, S., and Matsuzaka, Y. (1978), Cross-polarized radar backscatter from moist soil, Remote Sens. Environ. 7:211-217.

Jackson, T. J., and O'Neill, P. E. (1985), Aircraft scatterometer observations of soil moisture on rangelands watersheds, Int. J. Remote Sens. 6(7):1135-1152.

Le Toan, T., Pausader, M., Flouzat, G., and Fluhr, A. (1981), Soil moisture content and microwave backscatter in the $1.5-9 \mathrm{GHz}$ region, invited paper, Int. Geosci. Remote Sens. Symp., Washington, DC, June 1981.

Newton, R. W., Paris, J. F., and Clark, B. V. (1983), A microwave systems approach to measuring root zone soil moisture, NASA STAR (USA) Tech. Rep. 13, 69 pp.

Pausader, M. (1982), Contribution à la télédétection hyperfréquence: étude des relations entre la réflectivité radar et l'humi- dité du sol, thesis, Université Paul Sabatier, Toulouse, France.

Prevot, L., Bernard, R., Taconet, D., and Vidal-Madjar, D. (1984), Evaporation from a bare soil evaluated using a soil water transfer model and remotely sensed surface soil moisture data, Water Resour. Res. 20(2):311-316.

Ulaby, F. T. (1974), Radar measurement of soil moisture content, IEEE Trans. Antennas Propagation AP-22(2):257-265.

Ulaby, F. T., Batlivala, P. B., and Dobson, M. C. (1978), Microwave backscatter dependence on surface roughness, soil moisture, and soil texture: Part I-Bare soil, IEEE Trans. Geosci. Electron. GE16(4):286-295.

Wilheit, T. T. (1978), Radiative transfer in a plane stratified dielectric, IEEE Trans. $\dot{G} e-$ osci. Electron. GE-16(2):138-143.

Received 25 January 1988; revised 8 June 1988. 